\title{
EAl Endorsed Transactions

\section{Welcome message for the Special Issue "USCIAMO: Urban Sustainable, CollaboratIve, and Adaptive MObility"}

\author{
Silvia Gabrielli $^{1}{ }^{1}$, Stefania Castellani ${ }^{2}$, Antti Jylha ${ }^{3}$ and Jutta Willamowski ${ }^{2}$ \\ ${ }^{1}$ CREATE-NET, Trento, Italy Xerox \\ Research Centre Europe \\ ${ }^{3}$ University of Helsinki, Finland
}

\begin{abstract}
Transportation is a key domain to address for promoting sustainability as it accounts for about one third of the energy consumption in the EU and in the US. Nevertheless, changing the transportation habits of citizens is a hard challenge. In this Special Issue of the EAI Endorsed Transactions on Ambient Systems, we present a selection of high-quality papers presented at the workshop on "Urban Sustainable, CollaboratIve, and Adaptive MObility" (USCIAMO), held at the COOP 2014 Conference. The articles address different topics related to the design and deployment of innovative systems and techniques for behavior change in the domain of sustainable mobility, from gamification models and mechanics to encourage sustainable travel behavior to segmentation techniques for personalizing mobility behavior interventions, from participatory design of sustainable mobility applications to innovative frameworks for sustainable commuting at work and transport mode detection.
\end{abstract}

Keywords Urban Mobility, Sustainable Commuting, Game Mechanics, Behavior Change, Sustainable HCI, Persuasive Sustainability.

Received on 22 August 2014, published on 31 October 2014

Copyright (ㅇ 2014 Silvia Gabrielli et al., licensed to ICST. This is an open access article distributed under the terms of the Creative Commons Attribution licence (http://creativecommons.org/licenses/by/3.0/), which permits unlimited use, distribution and reproduction in any medium so long as the original work is properly cited.

doi: 10.4108/amsys.1.4.e1

\section{Introduction}

This Special Issue of the EAI Endorsed Transactions on Ambient Systems presents a selection of high quality papers presented at the workshop on "Urban Sustainable, CollaboratIve, and Adaptive MObility" (COOP 2014 Conference), updated and revised for final publication. The key objective of the workshop was to promote the gathering of researchers and practitioners active in the field of sustainable mobility design to promote the exchange of ideas and latest results in the context of the COOP 2014 Conference and to discuss new initiatives and systems that help travellers to improve the quality of their (sustainable) transportation. The workshop covered several topics related to gamification, profiling approaches and techniques for adapting sustainable mobility interventions, design studies for sustainable commuting and automatic mode detection.

Transportation represents a key domain to address for promoting sustainability as it accounts for about one third of the energy consumption in the EU and in the US. Nevertheless, changing the transportation habits of citizens

*Corresponding author. Email: silvia.gabrielli@ create-net.org is a hard challenge. Local governments have developed a wide variety of interventions to support sustainable mobility, in particular in urban settings. However, most of these typically do not reach the critical mass required to impact citizen's habits at a large scale. Nowadays, single occupancy vehicle (SOV) commuting still constitutes the main transportation means for most of the population. This induces high personal, societal and environmental costs, generating pollution, consuming energy and time, etc. To move a significant share of the population towards other, more sustainable urban transportation means, many aspects have to be considered, such as:

- The use of game mechanisms, gamification elements and incentives to motivate behavior change.

- Collaborative behaviors among travellers facilitating sustainable urban mobility; tools for collaboration and intercommunication between travellers.

- Effective design and assessment methods to refine and improve motivational functionalities for sustainable urban mobility over the long term. 
The first paper by Wells et al. presents a gamification model to encourage urban citizens to adopt more sustainable travel habits in daily life. The model has been implemented into a mobile journey planner and web platform realized during the SUPERHUB project, allowing citizens to track and reflect on their travel behavior, set challenges, and manage their progress towards sustainability related goals, which are expected to lead to the formation of desirable, long-term travel habits.

The paper authored by Millonig and Mitgutsch presents a framework for supporting the identification of game mechanics to be deployed to inform and motivate users towards sustainable mobility choices. In particular, the framework helps to systematically analyze group-specific effects of different game mechanics on mobility decision processes and inform the design of info-mobility services motivating users to explore unfamiliar, but more sustainable mobility options.

Forbes et al. describe a segmentation-based approach to design personalized behavior change interventions for sustainable, multi-modal urban mobility. The approach supports the delivery of personalized notifications based on specific user profiles. The paper reports results from a series of validation studies conducted by using Amazon Mechanical Turk ${ }^{\dagger}$ to obtain crowd-sourced categorization of motivational messages and deliver them to users in the context of the SUPERHUB project trials.

The paper by Bordin, Menendez and De Angeli presents the participatory design of ViaggiaTrento, a mobile application fostering sustainable mobility choices of student commuters in the city of Trento. The case study reports the requirements collected from students during the initial design phases of the application and the iterative refinement of the app functionalities to leverage from indications coming from the initial community of users to the rest of citizens.

Castellani et al. discuss the design of policy interventions in support of sustainable commuting. The paper addresses the requirements needed for designing technological solutions and services for commuters and work organizations that can comply with so-called "Workplace Travel Plans" and promote sustainable commuting among employees. The paper also describes a proposal for an IT infrastructure providing these services.

Finally, Biancat, Brighenti and Brighenti present a review of the state of the art in the field of transport mode detection techniques by focusing on solutions realized for different sensing devices and for mode classification. Results of a comparison among techniques are also provided, by highlighting current opportunities and challenges in this research and development field.
We are happy to present this Special Issue, and hope readers will enjoy it as much as we enjoyed participating and discussing with authors during the USCIAMO workshop in Nice.

We wish to thank all the speakers and participants of the workshop. Special thanks also to all reviewers, who provided a fundamental support to the realization and publishing of this Special Issue.

\footnotetext{
${ }^{\dagger}$ https://www.mturk.com/mturk/
} 